\title{
A influência da alimentação no tratamento da doença de alzheimer
}

\section{The influence of food in the treatment of alzheimer's disease}

\section{Carolina de Souza Balbino \\ Graduação em Nutrição \\ Faculdade Venda Nova do Imigrante - FAVENI \\ Rua Miguel Atta, $n^{\circ}$ 157, Morada do Castelo, Resende-RJ \\ carollina.balbino@gmail.com}

\section{RESUMO}

BALBINO, Carolina de Souza. A influência da alimentação no tratamento da doença de Alzheimer. 2019, 25 p. Monografia (graduação em Nutrição) Centro Universitário de Barra Mansa, Barra Mansa, RJ.

A doença de Alzheimer (DA) é um distúrbio neurodegenerativo causado pela deposição excessiva da proteína b-amilóide no cérebro. O b-amilóide é formado durante o processamentoda proteína precursora do amilóide, um composto que pode participar da manutenção da integridade e da regulação sináptica (JONES JR, 2006). Em geral, o primeiro aspecto clínico é a deficiência da memória recente, enquanto as lembranças remotas são preservadas até um certoestágio da doença. A doença de Alzheimer (DA) vem atingindo grande parte da população, elaacomete principalmente indivíduos a partir dos 65 anos. Pesquisas apontam que um estilo de vida mais saudável pode ajudar a reduzir o risco desta e outras demências. A influência dos aspectos nutricionais no processo de neurodegeneração vem sendo estudada desde sua participação protetora até a sua possível ação no retardo das disfunções e alterações degenerativas. Este estudo vem da demanda de oferecer informações para melhora da qualidadede vida de indivíduos acometidos pela doença de Alzheimer. Foi realizada uma revisãobibliográfica. Para tanto serão utilizadas como fonte de pesquisa livros, dissertações, teses e artigos científicos em sites especializados no período de 2005 ao ano corrente, com o objetivo de verificar a produção cientifica relacionada ao cuidado nutricional do paciente com Alzheimer. Estudos apontam que há ligações, ainda que não muito significativas, entre a alimentação e Alzheimer, são necessários mais estudos nesta área para que haja mais evidenciasque comprovem se essa ligação pode ser significativa ou não.

Palavras-chave: Alzheimer, nutrição.

\section{ABSTRACT}

BALBINO, Carolina de Souza. The influence of diet in the treatment of Alzheimer's disease. 2019, 25 p. Monograph (graduation in Nutrition) Centro Universitário de Barra Mansa, Barra Mansa, RJ.

Alzheimer's disease (AD) is a neurodegenerative disorder caused by the 
excessive composition of b-amyloid protein in the brain. B-amyloid is formed during processing of amyloid precursor protein, a compound that can participate in maintaining integrity and synaptic regulation (JONES JR, 2006). In general, the first clinical aspect is the most recent memory, while the memories removed are preserved until a certain stage of the disease. Alzheimer's disease (AD) has been affecting a large part of the population, being affected mainly from 65 years of age. Research indicates that a healthier lifestyle can help reduce or decrease risk and other dementias. The influence of nutritional aspects on the process of neurodegeneration has been studied since its protected participation until its possible action in the delay of dysfunctions and degenerative alterations. This study comes with the demand to provide information to improve the quality of life of patients with Alzheimer's disease. A literature review was performed. For this purpose, books, dissertations, theses and scientific articles will be used as a source of research in specialized websites from 2005 to the current year, with the purpose of verifying a useful scientific production for the nutritional care of Alzheimer's patients. Studies indicate that there are links, although not very significant, betweenfood and Alzheimer's disease, more studies are needed in this area, as there is more evidence that the link may be significant or not.

Keywords: Alzheimer's, nutrition.

\section{INTRODUÇÃO}

A doença de Alzheimer (DA) é um distúrbio neurodegenerativo causado pela deposição excessiva da proteína b-amilóide no cérebro. $\mathrm{O}$ b-amilóide é formado durante o processamento da proteína precursora do amilóide, um composto que pode participar da manutenção da integridade e da regulação sináptica (JONES JR, 2006).

Em geral, o primeiro aspecto clínico é a deficiência da memória recente, enquanto as lembranças remotas são preservadas até um certo estágio da doença. Além das dificuldades de atenção e fluência verbal, outras funções cognitivas deterioram à medida que a patologia evolui,entre elas a capacidade de fazer cálculos, as habilidades vísuoespaciais e a capacidade de usar objetos comuns e ferramentas. O grau de vigília e a lucidez do paciente não são afetados até a doença estar muito avançada. A fraqueza motora também não é observada, embora as contraturas musculares sejam uma característica quase universal nos estágios avançados da patologia (SERENIKI E VITAL, 2008).

Esses sintomas são frequentemente acompanhados por distúrbios comportamentais, como agressividade, alucinações, hiperatividade, irritabilidade e depressão. Transtornos do humor afetam uma porcentagem considerável de indivíduos que desenvolvem doença de Alzheimer, em algum ponto da evolução 
da síndrome demencial (SERENIKI E VITAL, 2008).

Em 2015, a população de idosos estimada no Brasil foi de 23 milhões, equivalendo a 12,5\%da população total de brasileiros (apud MATIOLI, 2016, p.2).

De acordo com o relatório de 2012 da Organização Mundial da Saúde (OMS), atualmente mais de 35 milhões de pessoas em todo o mundo têm demência, número que deveráduplicar em 2030 (66 milhões) e triplicar até 2050 (115 milhões). No Brasil, não existem dadosprecisos sobre quantas pessoas têm a DA, mas, segundo a OMS, estima-se que sejam aproximadamente 1,2 milhões de casos desta demência (WHO, 2012), já para o estado do RioGrande do Sul, a estimativa de pessoas com a demência passa dos 58 mil habitantes (COUTINHO FILHO, 2008).

O envelhecimento faz com que o ser humano busque cada vez mais mudanças em seusaspectos fisiológicos, e a nutrição está ligada em alterações nestas mudanças, o que pode tornar este envelhecimento saudável ou não (BUSNELLO et al, 2007).

A influência dos aspectos nutricionais no processo de neurodegeneração vem sendo estudada desde sua participação protetora até a sua possível ação no retardo das disfunções e alterações degenerativas (MACHADO et al.,2009)

Fatores de risco tais como hipertensão, dislipidemia, diabetes e obesidade parecem aumentar o risco do surgimento de demências em idosos por causarem danos ao tecido cerebraltais como estresse oxidativo, inflamação e disfunção endotelial (MIDDLETON e YAFFE, 2009).

\section{OBJETIVOS}

\subsection{OBJETIVO GERAL}

Verificar a produção cientifica relacionada ao cuidado nutricional do paciente comAlzheimer.

\subsubsection{Objetivos Específicos}

- Analisar a relação entre o desequilíbrio nutricional e o Alzheimer.

- Verificar como os nutrientes influenciam no tratamento e ou na prevenção. 


\section{REFERENCIAL TEÓRICO}

\subsection{A DOENÇA DE ALZHEIMER}

A doença de Alzheimer (DA) é um distúrbio neurodegenerativo causado pela deposição excessiva da proteína b-amilóide no cérebro. O b-amilóide é formado durante o processamento da proteína precursora do amilóide, um composto que pode participar da manutenção da integridade e da regulação sináptica (JONES JR, 2006).

Na maior parte dos casos, a DA manifesta-se inicialmente por uma perda insidiosa da memória seguida de uma demência lentamente progressiva. Esta patologia apresenta uma duração média entre 8 a 10 anos, estando associada a grande incapacidade. Apesar da maior parte dos indivíduos com doença de Alzheimer terem o mesmo tipo de sintomas e os efeitos serem semelhantes, a doença pode caracterizar-se em três categorias ou tipos diferentes.

As lesões de DA no SNC incluem placas de peptídeos $\beta(A \beta)$ amilóides e emaranhadosneurofibrilares da proteína tau hiperfosforilada, ambos ligados à perda de sinapse, morte neuronal e, finalmente, declínio cognitivo (Huang e Mucke, 2012).

De acordo com Sereniki e Vital (2008) essa doença é mais frequentemente associada à idade avançada, cujas manifestações cognitivas e neuropsiquiátricas resultam em deficiência progressiva e incapacitação. A doença afeta aproximadamente $10 \%$ dos indivíduos com idade superior a 65 anos e $40 \%$ acima de 80 anos.

Reitz et al. (2010) apud (Carretta e Scherer, 2012.) realizou um estudo longitudinal com1051 idosos de Nova York sem comprometimento demencial ou disfunção cognitiva no início da pesquisa. Verificou-se que os fatores de risco foram: idade, sexo, escolaridade, etnia, APOE épsilon4 genótipo, história de diabetes, hipertensão ou tabagismo, níveis de lipoproteína de altadensidade e relação cintura / quadril.

\subsubsection{Alzheimer de Início Precoce}

Alzheimer com início precoce é uma forma rara da doença (menos de 10\% de todas as pessoas com doença de Alzheimer têm início precoce). É considerado Alzheimer de início precoce quando a doença é diagnosticada antes dos 65 anos. 
Este tipo de doença é mais suscetível de se manifestar em pessoas com Síndrome de Down. Pensa-se que os genes que provocam o início precoce da doença são muito específicos e estão presentes no cromossomo 14 e 21 (CORREIA et al., 2015).

Fundamentalmente, o aparecimento do Alzheimer com início precoce é mais provável de ser marcado por sintomas atípicos, e as avaliações cognitivas apontam para pior funcionamento executivo e visuoespacial e praxia com prejuízo de memória menos acentuado. As características do tipo Alzheimer serão mais densas e difundidas em estudos de neuropatologia, com neuroimagem estrutural e funcional mostrando atrofia maior e mais difusaque se estende a áreas neocorticais (especialmente o precuneus) (TELLECHEA et al, 2018 apudESPIRITO-SANTO e DANIEL, 2019)

\subsubsection{Alzheimer de Início Tardio ou Alzheimer Esporádico}

A doença de Alzheimer de início tardio é a forma mais comum de aparecimento da doença sendo responsável por cerca de $90 \%$ dos casos. Geralmente ocorre depois dos 65 anos.Este tipo de doença de Alzheimer também pode ser designado por Alzheimer Esporádico e afetapessoas que podem ter ou não, antecedentes familiares da doença. Parece não existir hereditariedade na doença de Alzheimer Esporádico. Contudo, é possível que algumas pessoas possam herdar uma determinada suscetibilidade para desenvolver a doença numa idade avançada. O APOE (Apolipoproteína E) é o único gene associado a um ligeiro aumento do riscode desenvolver doença de Alzheimer de início tardio. Mesmo assim, metade das pessoas portadoras deste gene, e que vivem até aos 85 anos, não desenvolve demência nesta idade. (Nutrição e Doença de Alzheimer, 2015)

O risco de DA comum de início tardio está associado a variantes raras de receptores imunológicos expressos na microglia (Guerreiro e Hardy, 2014).

\subsubsection{ALZHEIMER FAMILIAR}

A doença de Alzheimer familiar é conhecida por ser inteiramente hereditária. É menos comum, sendo passada de uma geração para outra. Se um dos progenitores tem um gene mutado, cada filho terá $50 \%$ de probabilidade de 
herdá-lo. A presença do gene significa que existe possibilidade da pessoa desenvolver a doença de Alzheimer, normalmente entre os 40 e 60 anos. (CORREIA et al., 2015).

\subsubsection{RELAÇÃO ENTRE DIABETES MELLITUS E ALZHEIMER}

Diabetes mellitus integra um grupo de doenças metabólicas, ela ocorre pois as células de insulina por algum defeito na secreção não consegue utilizar de maneira eficiente a glicose ingerida (BRUNNER e SUDDARTH, 2006).

De acordo com Dallaqua e Damasceno (2009) apud (BARBOSA e CAMBOIM, 2016)o diabetes mellitus pode ser classificado em tipo 1 (DM1), tipo 2 (DM2) e gestacional. DM1 éa forma auto-imune, resulta da destruição das células pancreáticas por mecanismo mediado porcélulas. No DM2, os indivíduos afetados apresentam resistência à insulina, em combinação com deficiência relativa (não absoluta) da secreção de insulina, DM gestacional é caracterizado peloquadro de intolerância à glicose, com primeira identificação na gravidez e pode persistir apóso parto evoluindo para DM2.

Em contraste com o diabetes tipo 1, a maioria dos casos do tipo 2 diabetes são provocadas por fatores de estilo de vida e são portanto evitável; fazendo exercício, perdendo peso, corte de gordura e açúcares na dieta, reduzindo o consumo de álcool e parar ou evitar fumar reduzem o risco. Diabetes pode ser tratado com dieta, medicamentos orais, hipoglicemicos ou insulina. A alta prevalência de diabetes torna potencialmente um dos mais importantes fatores de risco modificáveis para demência (PRINCE et al.,2015).

A pesquisa sobre a relação entre o diabetes e a DA começou com o chamado "estudo Rotterdam", um estudo epidemiológico que investigou mais de 6000 idosos por dois anos e apontou uma correlação positiva entre a presença de diabetes mellitus e o desenvolvimento de Demência. Outro trabalho epidemiológico, mais recente, mostrou a incidência de aumento da DA em homens que ganharam peso entre os 30 e 45 anos de idade e em mulheres entre 30 e 45anos com índice de massa corpórea maior que 30. Já um estudo sueco apontou o aumento estatisticamente significativo do risco de homens que desenvolvem diabetes tipo 2 por volta dos 50 anos de idade de desenvolverem a DA. (FALCO et al., 2016.) 
Pesquisas mostraram que o diabetes tipo 2 na meia-idade está associado ao aumento dorisco de demência, doença de Alzheimer, demência vascular e comprometimento cognitivo. Naverdade, as pessoas que têm diabetes tipo 2 têm, em média, duas vezes mais chances de desenvolver demência em comparação com aquelas sem diabetes (ALZHEIMER SOCIETY OF CANADA, 2018).

\subsection{RELAÇÃO ENTRE HIPERTENSÃO ARTERIAL E ALZHEIMER}

A hipertensão arterial sistêmica (HAS) é uma condição clínica multifatorial caracteriza-se por níveis de pressão arterial. Está condição é frequentemente associada, a alterações funcionais e ou estruturais dos órgãos alvo como coração, encéfalo, rins e vasos sanguíneos e as alterações metabólicas, com aumento de risco para problemas cardiovasculares fatais e não fatais (ÁVILA et al., 2010).

Dentre as DCVs, a Hipertensão Arterial Sistêmica (HAS) constitui importante fator de risco para complicações cardíacas e cerebrovasculares, sendo considerada um problema de saúde pública em âmbito mundial. Em 2000, a prevalência da HAS na população mundial era de $25 \%$ e a estimativa para o ano de 2025 é de 29\%. Estudos realizados no Brasil revelaram que a prevalência da hipertensão variou entre 22,3 e 43,9\%, com média de 32,5\% (RADOVANOVIC et al, 2014).

Dentre as comorbidades presentes na DA, a hipertensão arterial sistêmica (HAS) foi a mais prevalente entre os idosos $66 \%$, independente da função cognitiva, seguida do diagnóstico de diabetes mellitus (DM) 33\%, hipercolesterolemia 33\% (ALZHEIMER SOCIETY OF CANADA, 2018).

A hipertensão diminui a integridade vascular da barreira sangue-cérebro, resultando emextravasamento de proteínas no tecido cerebral. Isso pode levar a danos celulares, uma reduçãona função neuronal ou sináptica, e apoptose (morte celular). Pode também aumentar acumulação de $A \beta$ insolúvel (beta-amilóide), contribuindo diretamente para a patologia da DA(PRINCE, 2014).

Em relação ao estilo de vida, como hábitos alimentares, aumento progressivo daprevalência de sobrepeso ou obesidade da população, à baixa adesão a realização de atividade física, contribui significativamente para esse quadro (WESCHENFELDER e MARTINI, 2012). 


\subsection{NUTRIÇÃO E ALZHEIMER}

A nutrição tem um papel muito importante nesta patologia, pois é capaz de evitar queo paciente fique ainda mais vulnerável e proporciona uma melhor qualidade de vida, retardando os sintomas e a progressividade da doença. A inclusão apropriada de nutrientes específicos pode apresentar um papel na proteção do paciente com DA, como é o caso da suplementação combinada de antioxidantes como a vitamina $\mathrm{C}$ e vitamina $\mathrm{E}$, que tem sido utilizada para reduzir a incidência do mal de Alzheimer. (MEDEIROS et al.,2016).

Tabela 1-Vitamina E

\begin{tabular}{|c|c|c|}
\hline \multicolumn{3}{|c|}{ Vitamina $\mathrm{E}$} \\
\hline $\begin{array}{l}\text { Doses Diárias } \\
\text { Recomendadas }\end{array}$ & Fontes Alimentares & Como atingir? \\
\hline $\begin{array}{l}\text { Homens e Mulheres } \\
\qquad \begin{array}{c}\text { (idade }>18 \text { ) } \\
15 \mathrm{mg} / \text { dia }\end{array}\end{array}$ & $\begin{array}{l}\text { Azeite, óleos de origem } \\
\text { vegetal, sementes de } \\
\text { girassol, frutos } \\
\text { oleaginosos (avelãs, } \\
\text { amêndoas, amendoins, } \\
\text { pinhões, nozes), creme } \\
\text { vegetal/margarina, }\end{array}$ & $\begin{array}{l}\text { - } 2 \text { Colheres de Sopa de Azeite }(20 \mathrm{~g})-2,8 \mathrm{mg} \\
\text { - } 30 \mathrm{~g} \text { sementes girassol- 7,4 mg } \\
\text { - } 30 \mathrm{~g} \text { amêndoas- } 7,2 \mathrm{mg} \\
\text { - } 30 \mathrm{~g} \text { avelãs- } 7,5 \mathrm{mg} \\
\text { - } 10 \mathrm{~g} \text { creme vegetal- } 1,5 \mathrm{mg}\end{array}$ \\
\hline
\end{tabular}

Programa Nacional para a Promoção da Alimentação Saudável Nutrição e Doença de Alzheimer, 2015

Burgener et al. (2008) apud (CARRETTA e SCHERER, 2012) revisaram 34 estudos nas áreas de restrição dietética, antioxidantes e dieta mediterrânea, os quais apresentaram provas de que as intervenções nutricionais contra a demência têm um grande potencial de

Prevenir o desenvolvimento da demência. A inclusão de frutas e vegetais, pão, trigo e outros cereais, azeite, peixe e vinho tinto pode reduzir o risco de Alzheimer. Algumas vitaminas, comoa B12, B3, C ou a B9, foram relacionadas em estudos ao menor risco de Alzheimer, embora outros estudos indiquem que não têm nenhum efeito significativo no início ou desenvolvimentoda doença e podem ter efeitos secundários. Algumas especiarias, como a cúrcuma e o açafrão,mostraram sucesso na prevenção da degeneração cerebral em ratos de laboratório. 
Tabela 1-Vitamina C

\begin{tabular}{|c|c|c|}
\hline \multicolumn{3}{|c|}{ Vitamina C } \\
\hline $\begin{array}{l}\text { Doses Diárias } \\
\text { Recomendadas }\end{array}$ & Fontes Alimentares & Como atingir? \\
\hline $\begin{array}{c}\text { Homem (idade }>18 \text { ) } \\
\qquad 90 \mathrm{mg} / \text { dia }\end{array}$ & $\begin{array}{l}\text { Citrinos (laranja, limão, } \\
\text { tangerinas e clementinas); } \\
\text { kiwis; morangos; legumes de } \\
\text { folha verde (couve } \\
\text { portuguesa, espinafres, } \\
\text { agrião); pimentos; brócolos; } \\
\text { couves de Bruxelas; batata. }\end{array}$ & $\begin{array}{l}\text { - } 2 \text { Kiwis }(140 \mathrm{~g})-100 \mathrm{mg} \text {; } \\
\text { - } 1 \text { Laranja média }(160 \mathrm{~g})-91 \mathrm{mg} \text {; } \\
\text { - } 10 \text { Morangos }(150 \mathrm{~g})-71 \mathrm{mg} ; \\
\text { - } 100 \mathrm{~g} \text { couve portuguesa- } 58 \mathrm{mg} \\
\text { - } 100 \mathrm{~g} \text { couve-lombarda }-44 \mathrm{mg} \\
\text { - } 1 / 4 \text { Pimento }(40 \mathrm{~g})-43 \mathrm{mg} \\
\text { - } 6 \text { Brócolos }(180 \mathrm{~g})-32 \mathrm{mg} \text {. }\end{array}$ \\
\hline
\end{tabular}

Programa Nacional para a Promoção da Alimentação Saudável Nutrição e Doença de Alzheimer, 2015

Duron e Hanon (2008) apud (BUENO, 2014) relataram que o dano a células endoteliaisdos vasos sanguíneos cerebrais gera um estresse oxidativo com aumento da permeabilidade dabarreira hematoencefálica para proteínas, levando à acumulação da $\beta$-amiloide. Devido a essa relação de estresse oxidativo e demência, pesquisadores têm trabalhado com a hipótese de que alimentos antioxidantes podem retardar o déficit cognitivo e diminuir o risco para demências.

Diferentes tipos de estudos epidemiológicos têm permitido acumular informação sobre os efeitos positivos dos ácidos graxos ómega 3 e micronutrientes como as vitaminas do complexo $B$, vitaminas $E, C$ e $D$ sobre os neurónios (PRINCE et al.,2015). 
Tabela 1-Vitamina D

\begin{tabular}{|c|c|c|}
\hline \multicolumn{3}{|c|}{ Vitamina D } \\
\hline $\begin{array}{c}\text { Doses Diárias } \\
\text { Recomendadas }\end{array}$ & Fontes Alimentares & Como atingir? \\
\hline $\begin{array}{l}\text { Homens e Mulheres } \\
\qquad \begin{array}{c}\text { (idade } 19-70 \text { ) } \\
15 \mu g / \text { dia } \\
\text { (idade> 70) } \\
20 \mu g / \text { dia }\end{array}\end{array}$ & $\begin{array}{l}\text { Peixe (sardinha, corvina, } \\
\text { cherne, truta, salmão, } \\
\text { dourada, enguia, linguado); } \\
\text { óleos de figado de peixe; } \\
\text { gema de ovo; leite } \\
\text { enriquecido em cálcio ou } \\
\text { leite de crescimento; } \\
\text { margarinas vegetais e } \\
\text { cereais de pequeno-almoço } \\
\text { enriquecidos. }\end{array}$ & 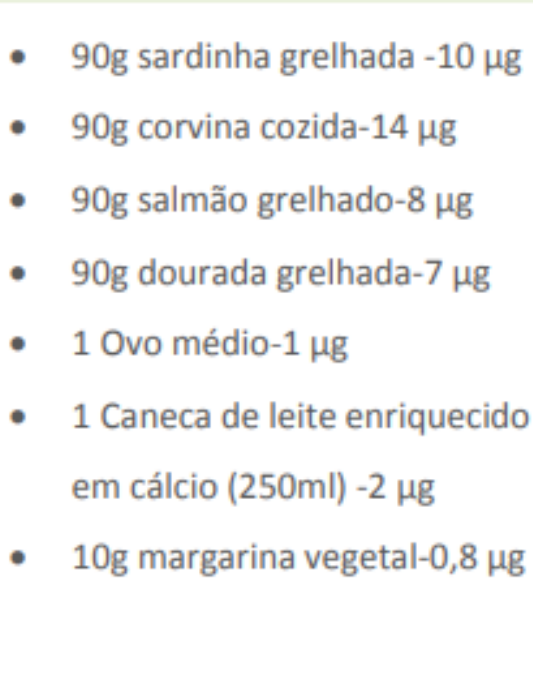 \\
\hline
\end{tabular}

Programa Nacional para a Promoção da Alimentação Saudável Nutrição e Doença de Alzheimer, 2015

Nutrientes como ácido fólico e ômega 3 podem ajudar na determinação da quantidade do caráter e funcionamento de neurotransmissores que alteram os processos cerebrais. Estudos mostram que pessoas com dietas ricas em colesterol e gorduras saturadas, e baixa quantidade de fibras, frutas e vegetais, têm uma maior chance de desenvolvimento de DA, porém, com umaalimentação equilibrada, rica em fibras, vitamina B12, B6 e ômega-3, a chance do desenvolvimento de doenças degenerativas diminui. (MEDEIROS et al., 2016). 


\begin{tabular}{|c|c|c|}
\hline $\begin{array}{l}\text { Doses Diárias } \\
\text { Recomendadas }\end{array}$ & Fontes Alimentares & Como atingir? \\
\hline $\begin{array}{c}\text { Homem } \\
\text { (idade 19-50) } \\
1,3 \mathrm{mg} / \text { dia } \\
\text { (idade> 50) } \\
1,7 \mathrm{mg} / \text { dia } \\
\text { Mulher } \\
\text { (idade } 19-50 \text { ) }\end{array}$ & $\begin{array}{l}\text { Carne, peixe, visceras, } \\
\text { gema de ovo, } \\
\text { leguminosas secas, alguns } \\
\text { tipos de queijo, cereais } \\
\text { integrais, flocos de } \\
\text { cereais, batata, alho } \\
\text { francês, hortícolas de } \\
\text { folha verde, couve-flor, } \\
\text { banana, frutos } \\
\text { oleaginosos. }\end{array}$ & $\begin{array}{l}2 \text { Batatas médias }(140 \mathrm{~g})-0,5 \mathrm{mg} \\
100 \mathrm{~g} \text { alho-francês- } 0,5 \mathrm{mg} \\
6 \text { Florescências de couve-flor }(180 \mathrm{~g}) \text { - } \\
0,3 \mathrm{mg} \\
1 \text { Banana média }(130 \mathrm{~g})-0,4 \mathrm{mg} \\
90 \mathrm{~g} \text { de truta grelhada- } 0,3 \mathrm{mg} \\
1 \text { Ovo médio- } 0,2 \mathrm{mg} \\
3 \text { Colheres de sopa de grão-de-bico }(80 \mathrm{~g} \\
-0,1 \mathrm{mg}\end{array}$ \\
\hline
\end{tabular}

Programa Nacional para a Promoção da Alimentação Saudável Nutrição e Doença de Alzheimer, 2015

Tabela 1-Omega 3

\begin{tabular}{|c|c|c|}
\hline \multicolumn{3}{|c|}{ Ácidos Gordos Ómega 3} \\
\hline $\begin{array}{l}\text { Doses Diárias } \\
\text { Recomendadas }\end{array}$ & Fontes Alimentares & Como atingir? \\
\hline $\begin{array}{l}\text { Homem (idade> 18) } \\
\qquad 1,6 \mathrm{~g} / \text { dia }\end{array}$ & $\begin{array}{l}\text { Peixes gordos (sardinha, cavala, } \\
\text { salmão e atum); óleos de peixe; } \\
\text { algas; nozes; sementes de linhaça e } \\
\text { de chia; óleos vegetais (noz, soja, } \\
\text { canhola e de linho); beldroegas; } \\
\text { espinafres; alho francês; couves; } \\
\text { cogumelos; margarinas vegetais } \\
\text { enriquecidas e ovos enriquecidos. }\end{array}$ & $\begin{array}{l}\text { - } 90 \mathrm{~g} \text { sardinha }-4,5 \mathrm{~g} \\
\text { - } 90 \mathrm{~g} \text { salmão grelhado-3,9g } \\
\text { - } 90 \mathrm{~g} \text { cavala-3,7g } \\
\text { - } 90 \mathrm{~g} \text { dourada grelhada-2g } \\
\text { - } 30 \mathrm{~g} \text { nozes-2,7 } \\
\text { - } 10 \mathrm{~g} \text { sementes de linhaça-2,3g } \\
\text { - } 10 \mathrm{~g} \text { sementes de chia-2g }\end{array}$ \\
\hline
\end{tabular}

Programa Nacional para a Promoção da Alimentação Saudável Nutrição e Doença de Alzheimer, 2015 


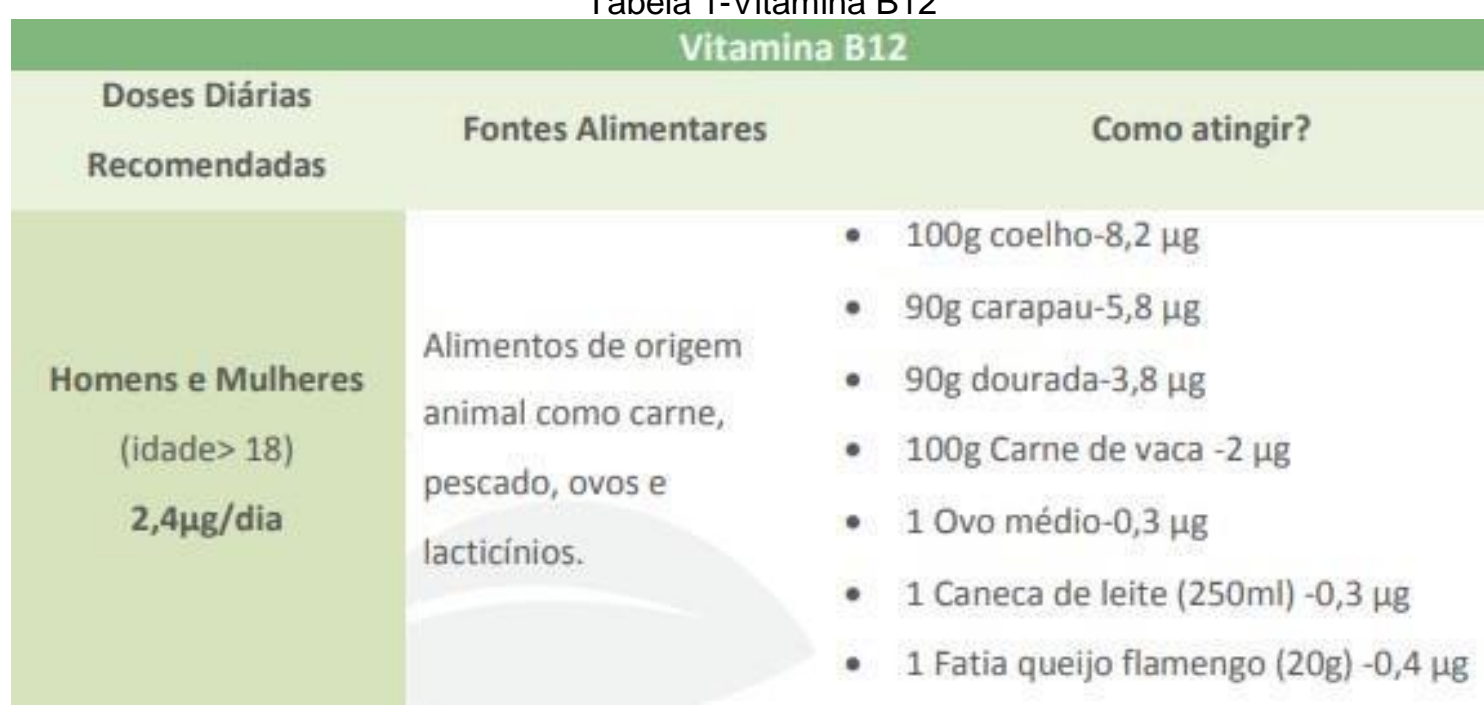

Programa Nacional para a Promoção da Alimentação Saudável Nutrição e Doença de Alzheimer, 2015

Muitos processos dependentes de tiamina (vitamina B1) estão diminuídos em cérebros de pacientes com DA. Karuppagounder et al., em seu estudo, demonstraram que a deficiência de tiamina exacerbou placas amilóides em ratos transgênicos, aumentou a área ocupada por placas no córtex, hipocampo e tálamo, e induziu inflamação em áreas formadoras de placa. A ingestão de vitamina B3 da dieta está inversamente associada à DA, tendo um efeito protetor no desenvolvimento da doença e no declínio cognitivo (GREGORIO et al., 2019).

Estudos relatam que pacientes com DA apresentam deficiências nutricionais de muitasvitaminas e minerais. As deficiências de micronutrientes podem resultar não apenas na perda de massa magra, declínio das funções imunológicas e aumento do risco de fraturas, mas tambémno dano oxidativo no cérebro e deficiências nos neurotransmissores, prejudicando a função cognitiva (GREGORIO et al., 2019).

Para idosos portadores de DA não avançada caracterizada pela falta de autonomia em suas atividades rotineiras, o estabelecimento das necessidades energéticas é importante, pois essa população é com frequência desnutrida. Essa situação ainda não está clara se é decorrentede uma taxa metabólica de repouso aumentada ou se é o maior gasto de energia que causa a perda de peso. Para outros estudiosos a alimentação é negligenciada e a ingestão alimentar inadequada resulta em menor independência e a auto-alimentação ineficiente (MAHAN et al. 2012). 
Deve-se garantir uma dieta adequada ao portador do Mal de Alzheimer, que inclua proteínas e calorias extras de acordo com idade, sexo e atividade. Pessoas com Alzheimer podem requerer $35 \mathrm{kcal} / \mathrm{kg}$ de peso corporal (RÉQUIA e OLIVEIRA, 2006).

A dieta dos pacientes deve conter níveis mais elevados de proteína e menor carboidratodesde o início do tratamento da doença, particularmente para manter os níveis de neurotransmissores como serotonina, adrenalina e dopamina. Os neurotransmissores precisam de alguns precursores, como triptofano, colina e tirosina, que estão presentes nos alimentos proteico (GREGORIO et al., 2019).

\section{METODOLOGIA}

Foi realizada uma revisão bibliográfica. Para tanto foram utilizadas como fonte de pesquisa livros, dissertações, teses e artigos científicos em sites especializados datados de 2005 ao ano corrente, com o objetivo de avaliar a produção cientifica relacionada aos possíveis efeitos da alimentação e nutrição no tratamento da doença de Alzheimer. A pesquisa foi realizada por meio de consultas às bases de dados Medline, Scientific Eletronic Library On-line (SCIELO), Scholar Google (Google Acadêmico), Pubmed e Biblioteca virtual de saúde (Bvs). Para isso, foram utilizados os seguintes descritores: Alzheimer; nutrição.

Foram considerados relevantes para o estudo o total de 26 produções científicas, oferecendo evidências disponíveis relacionada ao cuidado nutricional do paciente com Alzheimer.

\section{CONSIDERAÇÕES FINAIS}

O desenvolvimento do presente estudo avaliou como a alimentação pode influenciar notratamento da doença de Alzheimer.

Foi observado que fatores que se modificam com a alimentação, como diabetes mellitustipo 2 e hipertensão arterial, podem influenciar na progressão da DA, fazendo com que os processos de neurodegeneração avancem mais rapidamente.

Deficiências nutricionais de vitaminas e minerais aumentam o risco de 
dano oxidativo no cérebro e também causam problemas nos neurotransmissores, prejudicando a função cognitiva.

A inclusão apropriada de vitaminas antioxidantes como $C$ e $E$, e também as vitaminas do complexo $\mathrm{B}$, podem apresentar um papel na proteção do paciente com DA.

O ômega 3 também apresentou ação protetora em algumas pesquisas.

A pratica exercícios fisicos, manter o peso adequado, uma alimentação saudavel, reduziro consumo de álcool e parar ou evitar fumar, ajudam a reduzir o risco para a DA.

Indivíduos portadores de DA normalmente encontra-se desnutrida. Não foi possível determinar se esta é decorrente de uma taxa metabólica de repouso aumentada ou se é o maiorgasto de energia que causa a perda de peso.

Deve conter níveis mais elevados de proteínas na alimentação dos indivíduos com DA pois, os neurotransmissores necessitam de alguns aminoácidos precursores que estão presentesnesses alimentos.

Estudos apontam que há ligações, ainda que não muito significativas, entre a alimentação e Alzheimer, são necessários mais estudos nesta área para que haja mais evidenciasque comprovem essa ligação 


\section{REFERÊNCIAS}

Middleton, L.E.; Yaffe, K. Promising Strategies for the Prevention of Dementia. Archives of Neurology, 66,1210-1215.2009.Disponível em $<$ http://dx.doi.org/10.1001/archneurol.2009.201> Acesso em; 13 out. 2018

Alzheimer Society of Canada. Alzheimer fatores de risco. Disponível em:

$<$ http://alzheimer.ca/en/Home/About-dementia/Alzheimer-s-disease/Riskfactors $>$. Acesso em: 13 out. 2018.

ÁVILA et al. VI Diretrizes Brasileiras de Hipertensão. I Diretrizes Brasileiras de Hipertensão. Arq. Bras. Cardiol. São Paulo, v. 95, n. 1. p.4, 2010.Disponível em:

$<$ http://publicacoes.cardiol.br/consenso/2010/Diretriz_hipertensao_associados.p df> Acesso:em: 13 out. 2018.

BARBOSA, S. A.; CAMBOIM, F. E. F. Diabetes mellitus: cuidados de enfermagem para controle e prevenção de complicações. Temas em Saúde. v. 16, n. 3, 2016.

BORGHI, Ana Carla et al. Qualidade de vida de idosos com doença de Alzheimer e de seus cuidadores. Revista Gaúcha de Enfermaria. Porto Alegre, v. 32, n. 4, p. 751-758. Dec. 2011.

BUSNELLO, F. Aspectos Nutricionais No Processo Do Envelhecimento. São Paulo:Atheneu, 2007. 292p.

CARRETTA, M.; SCHERER, S. Perspectivas atuais na prevenção da doença de Alzheimer.

Estudos Interdisciplinares sobre o Envelhecimento, Porto Alegre, v. 17, n. 1, p. 3757, 2012.

CECÍLIO, A; OLIVEIRA, J. Educação Nutricional Para Idosos Intitucionalizados no Recanto Nossa Senhora do Rosário em Limeira, SP. Estudo Interdisciplinar do envelhecimento. PortoAlegre, v. 20, n. 2, p. 413-426, 2015

CORREIA, A; FILIPE, J; SANTOS, A; GRAÇA, P. Nutrição e Doença de Alzheimer. Disponível em: $<$ https://www.alimentacaosaudavel.dgs.pt/activeapp/wpcontent/files_mf/1444910422Nutri\%C3\%A7\%C3\%A3oeDoen\%C3\%A7adeAlzhei mer.pdf> Acesso: em: 13 out. 2018.

COUTINHO, C. As influências da prática de atividade física nas funções cognitivas em idosos.

Lecturas: Educación Física y Deportes, Buenos Aires. n. 118, p. 1-1, 2008.

ESPIRITO-SANTO, H; DANIEL. Trajetos do Envelhecimento Perspectivas

Teóricas eEmpíricas. Coimbra: imprensa universidade de Coimbra, 2019. 395p. 
FALCO, A. et al. Doença de Alzheimer: hipóteses etiológicas e perspectivas de tratamento. Quím. Nova, São Paulo,v. 39,

n. 1, p. 63-80, Jan. 2016. Disponível em

<http://www.scielo.br/scielo.php?script=sci_arttext\&pid=S0100

- 40422016000100063\&lng=en\&nrm=iso >. access on 19 Nov.

2019.

GREGORIO, E. et al. Nutritional and hematological factors associated with the progression ofAlzheimer's disease: a cohort study. Revista Associação Medica Brasileira. São Paulo, v.65,

n.2.p.222-231, fev.2019 Disponível em $<$ http://www.scielo.br/scielo.php?script=sci_arttext\&pid=S0104423020190002002 22\&lng=pt \&nrm=iso>. Acesso em 30 jun. 2019.

GUERREIRO R. HARDY J. Genetics of Alzheimer's disease. Neurotherapeutics. 2014. HUANG, Y.; MUCKE, L. Alzheimer mechanisms and therapeutic strategies. Cell. SanFrancisco, p. 1204-1222, 2012.

JONES JR, H. HOYDEN. Neurologia de Netter.1.ed.Porto Alegre: Artmed, 2006. $1008 \mathrm{p}$.

MACHADO, J. et al. Estado nutricional na doença de Alzheimer. Revista Associação Medica

Brasileira. v.55, n.2, p.188-191, 2009.

MAHAN, L. K.; ESCOTT- STUMP, S. Alimentos nutrição \& dietoterapia. 13. Ed. Rio deJaneiro, 2012.

MATIOLI, M. Associação entre diabetes mellitus e demência: estudo neuropatológico. 2016. Tese (Doutorado em Neurologia) - Faculdade de Medicina, Universidade de São Paulo, São Paulo, 2016. doi:10.11606/T.5.2016.tde-18112016-111336. Acesso em: 03 set. 2018.

MEDEIROS, G. et al. Perfil nutricional de idosos portadores de Alzheimer atendidos em homecare. Revista Brasileira de Neurologia. v.52 n.4. Out/Nov/Dez 2016.

PRINCE, M. et al. Dementia and Risk Reduction. Alzheimer's Disease International. London, Sept. 2014.

PRINCE, M. et al. The global impact of dementia. Alzheimer's Disease International. London, Oct. 2015.

RADOVANOVIC, C.A; SANTOS, L; CARVALHO, M.D; MARCON, S. Hipertensão arterial

e outros fatores de risco associados às doenças cardiovasculares em adultos.

Rev. Latino-Am.Enfermagem, São Paulo, v.22 n.4 p. 547-553, jul.-ago. 2014.

RÉQUIA, C.; OLIVEIRA, V. Aspectos Fisiológicos e Nutricionais na Doença de Alzheimer.

Revista Nutrição em Pauta, São Paulo, n.80. Out. 2006. 
SERENIKI, A; VITAL, M.A. A doença de Alzheimer: aspectos fisiopatológicos e farmacológicos. Revista de Psiquiatria do Rio Grande do Sul, Rio Grande do Sul, v.30 n.1 p. 2008.

SILVA, S; OLIVEIRA, S; PIERIN, A.M. O controle da hipertensão arterial em mulheres e homens: uma análise comparativa. Arquivo Brasileiro de Cardiologia. vol.95 no.1 supl.1 SãoPaulo, 2010.

WESCHENFELDER MAGRINI, D; GUE MARTINI, J. Hipertensão arterial: principais fatoresde risco modificáveis na estratégia saúde da família. Revista Electrónica Trimestral de Emfermaria. 